\title{
The new solar abundances - Part I: the observations
}

\author{
M. Asplund ${ }^{1}$, N. Grevesse ${ }^{2,3}$ and A.J. Sauval ${ }^{4}$ \\ ${ }^{1}$ Research School of Astronomy and Astrophysics, Australian National University, \\ Cotter Road, Weston 2611, Australia \\ ${ }^{2}$ Centre Spatial de Liège, Université de Liège, Avenue Pré Aily, B-4031 Angleur-Liège, Belgium \\ ${ }^{3}$ Institut d'Astrophysique et de Géophysique, Université de Liège, \\ Allée du 6 Août, 17, B5c, B-4000 Liège Belgium \\ ${ }^{4}$ Observatoire Royal de Belgique, Avenue Circulaire, 3, B-1180 Bruxelles, Belgium
}

\begin{abstract}
The new solar abundances have been derived from analyses of the photospheric spectrum. They result from the use of a 3D hydrodynamical model of the solar atmosphere instead of the classical 1D hydrostatic models, accounting for departures from LTE when possible and improved atomic and molecular data. The new solar abundances are lower than previously recommended values and the present solar metallicity, $Z$, and $Z / X$, decrease to $Z=0.0122$ and $Z / X=0.0165$ respectively, almost a factor of two lower than earlier widely used values. We briefly discuss the new results for the most abundant elements, show why they are trustworthy and discuss some implications (see also Montalbán et al. 2006, Part II).
\end{abstract}

\section{Introduction}

New generation of three-dimensional (3D) hydrodynamical models of the solar lower atmosphere have been applied, for the first time, to the analysis of the solar photospheric spectrum, instead of the classical 1D photospheric models used during more than four decades. These new 3D models combined with calculations of non-LTE effects, when possible, and the use of improved atomic and molecular data, lead to significant downward revisions of the abundances of the most abundant elements, $\mathrm{C}, \mathrm{N}, \mathrm{O}, \mathrm{Na}$ to $\mathrm{Ca}$, Fe as well as $\mathrm{Ne}$ and $\mathrm{Ar}$ (Asplund et al. 2005a). We shall briefly discuss the new results, show why they are really trustworthy and discuss some implications (see also Montalbán et al. 2006, Part II).

\section{New results}

\section{$2.1 \mathrm{C}, \mathrm{N}$ and $\mathrm{O}$}

The new solar abundances of $\mathrm{C}, \mathrm{N}$ and $\mathrm{O}$ have been derived from a large variety of abundance indicators, forbidden and permitted lines as well as various molecular lines in order to minimize systematic errors. We have also been very demanding on the quality of the lines. We also carried out detailed non-LTE calculations when the required atomic data were available. Detailed accounts of our new analyses have recently been published (Asplund et al. 2004, 2005a, 2005b). Results are shown in Table 1 where they are compared with similar results obtained with the widely used 1D hydrostatic photospheric model of Holweger and Müller (1974). 
The new solar abundances of $\mathrm{C}, \mathrm{N}$ and $\mathrm{O}$ are $\epsilon_{C}=8.39, \epsilon_{N}=7.78, \epsilon_{O}=8.66$ respectively, in the usual logarithmic scale relative to hydrogen. These new values are much lower than those recommended in widely used compilations : $-0.17 \operatorname{dex}(\mathrm{C}),-0.27 \operatorname{dex}(\mathrm{N}$ and $\mathrm{O}$ ) when compared with Anders and Grevesse (1989) and -0.13 dex (C), -0.14 dex $(\mathrm{N})$ and $-0.17 \operatorname{dex}(\mathrm{O})$ if we refer to more recent reviews by Grevesse and Noels (1993) and Grevesse and Sauval (1998).

Table 1: C, N, O abundances as implied from a variety of different atomic and molecular indicators using a 3D hydrodynamical model of the solar atmosphere (Asplund et al. 2005a). Results from the semi-empirical 1D model of Holweger-Müller (1974) are given for comparison. The last column gives the difference between 3D- and 1D-based results

\begin{tabular}{|l|c|c|r|}
\hline \multirow{2}{*}{ Lines } & \multicolumn{3}{|c|}{ CNO abundances } \\
\cline { 2 - 4 } & $3 \mathrm{D}$ & $\mathrm{HM}$ & 3D-1D \\
\hline$[\mathrm{CI}]$ & 8.39 & 8.45 & -0.06 \\
$\mathrm{CI}$ & $8.36 \pm 0.03$ & $8.39 \pm 0.03$ & -0.03 \\
$\mathrm{CH} \Delta v=1$ & $8.38 \pm 0.04$ & $8.53 \pm 0.04$ & -0.15 \\
$\mathrm{C}_{2}$ Swan & $8.44 \pm 0.03$ & $8.53 \pm 0.03$ & -0.09 \\
$\mathrm{CH} \mathrm{A}-\mathrm{X}$ & $8.45 \pm 0.04$ & $8.59 \pm 0.04$ & -0.14 \\
$\mathrm{CO} \Delta v=1$ & $8.41 \pm 0.02$ & $8.62 \pm 0.02$ & -0.21 \\
$\mathrm{CO} \Delta v=2$ & $8.38 \pm 0.02$ & $8.70 \pm 0.03$ & -0.32 \\
\hline $\mathrm{NI}$ & $7.85 \pm 0.08$ & $7.97 \pm 0.08$ & -0.12 \\
$\mathrm{NH} \Delta v=1$ & $7.73 \pm 0.05$ & $7.95 \pm 0.05$ & -0.22 \\
\hline$[\mathrm{OI}]$ & $8.68 \pm 0.01$ & $8.76 \pm 0.02$ & -0.08 \\
$\mathrm{OI}$ & $8.64 \pm 0.02$ & $8.64 \pm 0.08$ & 0.00 \\
$\mathrm{OH} \Delta v=0$ & $8.61 \pm 0.03$ & $8.82 \pm 0.01$ & -0.21 \\
$\mathrm{OH} \Delta v=1$ & $8.61 \pm 0.03$ & $8.87 \pm 0.03$ & -0.26 \\
\hline
\end{tabular}

\section{$2.2 \mathrm{Ne}$ and $\mathrm{Ar}$}

No spectral lines of $\mathrm{Ne}$ and $\mathrm{Ar}$ are present in the photospheric spectrum. Their abundances are estimated from the abundance ratios, $\mathrm{Ne} / \mathrm{O}$ and $\mathrm{Ar} / \mathrm{O}$, measured in different types of coronal matter, with the photospheric abundance for $\mathrm{O}$. The $\mathrm{Ne}$ and $\mathrm{Ar}$ abundances, $\epsilon_{\mathrm{Ne}}=$ 7.84 and $\epsilon_{A r}=6.18$ respectively, are therefore directly affected by the revised solar oxygen abundance and are much lower than the values previously recommended in the compilations cited hereabove.

Very recently, Drake and Testa (2005) suggested that a higher $\mathrm{Ne} / \mathrm{O}=0.4$, compared to 0.15 adopted in our analysis, might be appropriate for the Sun, from the analyses of the coronae of very highly active stars. We believe that these very highly active stars do not represent real solar matter. A large number of solar measurements relative to active centers and quiet corona lead to low values of the $\mathrm{Ne} / \mathrm{O}$ ratio. The very recent solar analyses by Schmelz et al. (2005) and Young (2005) confirm these low ratios. Furthermore the $\mathrm{Ne} / \mathrm{O}$ ratios measured in the local galactic medium, hot stars, HII regions and planetary nebulae are also in good agreement with the low value. For all these reasons, we believe the true solar $\mathrm{Ne} / \mathrm{O}$ ratio to be 0.15 , the value we adopted.

\subsection{Intermediate elements ( $\mathrm{Na}$ to $\mathrm{Ca}, \mathrm{Fe}$ )}

$3 \mathrm{D}$ analyses of $\mathrm{Na}, \mathrm{Mg}, \mathrm{Al}, \mathrm{Si}, \mathrm{P}, \mathrm{S}, \mathrm{K}, \mathrm{Ca}$ and $\mathrm{Fe}$ have also been performed. When possible, departures from LTE have been taken into account. Results are given in Table 2 where they 
are compared with 1D-based values. The new 3D-based abundances are smaller than the $1 \mathrm{D}$-based results but the impact of the 3D model atmosphere is smaller than for CNO. The actual decreases are of order 0.05 to $0.10 \mathrm{dex}$.

Table 2: $\mathrm{Na}$ to $\mathrm{Ca}$ and Fe abundances as implied from a variety of different atomic indicators using a 3D hydrodynamical model of the solar atmosphere (Asplund et al. 2005a). Results from the semi-empirical 1D model of Holweger-Müller (1974) are given for comparison. The last column gives the difference between 3D- and 1D-based results

\begin{tabular}{|l|c|c|c|}
\hline \multirow{2}{*}{ Elts } & \multicolumn{3}{|c|}{$\mathrm{Na}$ to Ca, Fe abundances } \\
\cline { 2 - 4 } & 3D & HM & 3D-1D \\
\hline NaI & $6.17 \pm 0.04$ & $6.27 \pm 0.04$ & -0.10 \\
\hline $\mathrm{MgI}$ & $7.57 \pm 0.23$ & $7.64 \pm 0.23$ & -0.07 \\
$\mathrm{MgII}$ & $7.53 \pm 0.08$ & $7.56 \pm 0.08$ & -0.03 \\
\hline $\mathrm{AlI}$ & $6.37 \pm 0.06$ & $6.45 \pm 0.06$ & -0.08 \\
\hline $\mathrm{SiI}$ & $7.51 \pm 0.04$ & $7.55 \pm 0.04$ & -0.04 \\
$\mathrm{SiII}$ & 7.45 & 7.46 & -0.01 \\
\hline $\mathrm{PI}$ & $5.36 \pm 0.04$ & $5.37 \pm 0.04$ & -0.01 \\
\hline $\mathrm{SI}$ & $7.14 \pm 0.05$ & $7.17 \pm 0.05$ & -0.03 \\
\hline $\mathrm{KI}$ & $5.08 \pm 0.07$ & $5.20 \pm 0.07$ & -0.12 \\
\hline $\mathrm{CaI}$ & $6.30 \pm 0.04$ & $6.43 \pm 0.04$ & -0.13 \\
$\mathrm{CaII}$ & $6.32 \pm 0.08$ & $6.34 \pm 0.08$ & -0.02 \\
\hline FeI & $7.44 \pm 0.05$ & $7.50 \pm 0.05$ & -0.06 \\
FeII & $7.45 \pm 0.10$ & $7.47 \pm 0.10$ & -0.02 \\
\hline
\end{tabular}

\section{Why are the new abundances trustworthy ?}

We summarize hereafter various arguments in favour of the new solar abundance results. More details can be found in recent reviews (Asplund 2005; Asplund et al. 2005a; Grevesse et al. 2005).

The use of a 3D hydrodynamical model represents a real step forward in the modelling of the inhomogeneous solar atmosphere. Without any free parameter, these models successfully reproduce various key observational diagnostics whereas 1D models obviously fail : granulation properties, topology and statistics, some helioseismological constraints, the emergent flux distribution and limb-darkening, the intensity brightness contrast and, last but not least, the shapes, shifts and asymmetries of the photospheric spectral lines (see Fig. 8 of Asplund et al. 2000). For the first time, we are able to fit nearly perfectly a predicted line profile with the observed one and we don't rely anymore on fudge parameters like micro- and macroturbulence, needed with $1 \mathrm{D}$ models, to reproduce the widths of the line profiles.

Non-LTE effects in F-, G- and K-type stars have very recently been reviewed by Asplund (2005). Even if the non-LTE effects are rather small in the Sun, they nevertheless play an important role for a few important elements considered here (Asplund et al. 2004; Asplund et al. 2005b).

The combined 3D plus non-LTE effects on the abundances are clearly visible in Table 1 especially in the cases of $C$ and $O$ which have the largest number of indicators, formed in very different layers of the solar photosphere and with very different sensitivities to temperature and non-LTE. The excellent agreement of the 3D-based results for the various indicators is one additional important argument in favour of our new results. In contrast the 1D-based results show very large dispersions $(0.2-0.3 \mathrm{dex})$ among the various indicators and large differences 
with the 3D-based results, especially for the very sensitive molecular species. Furthermore 3D non-LTE treatments are needed to explain the center-to-limb behaviour of the Ol triplet at $777 \mathrm{~nm}$.

In addition, the new lower CNO solar abundances are now in excellent agreement with the values measured in the interstellar medium, in nearby B stars. The Sun has returned to normal : it is a Sun-like star.

\section{Summary and conclusions}

With the new solar chemical composition (see Asplund et al. 2005b and Grevesse et al. 2005, for more details and a complete revised table with the new solar chemical composition) the present $\mathrm{X}, \mathrm{Y}$ and $\mathrm{Z}$ become $\mathrm{X}=0.7393, \mathrm{Y}=0.2485$ and $\mathrm{Z}=0.0122$ with $\mathrm{Z} / \mathrm{X}=0.0165$. The abundance of helium adopted is obtained from inversion of helioseismic data by Basu and Antia (2004; see also Montalbán et al. 2006, Part II). The new metallicity and Z/X are much lower than the previously recommended values. The widely used metallicity $Z=0.02$ should be definitely forgotten and replaced by the new value, $Z=0.0122$.

Protosolar abundances are somewhat larger because of the effects of diffusion at the bottom of the convective zone : $Z_{0}=0.0132$ and $Z_{0} / X_{0}=0.0185$ (Grevesse et al. 2005).

Stellar abundances, at least for F-, G- and K-type stars, are also very much affected by the problems of the inhomogeneities in their outer layers and the non-LTE effects (Asplund 2005). This has to be kept in mind when comparing stellar and solar abundances.

The only problem with the new solar abundances is described in Part II of this paper by Montalbán et al. (2006). The question is : is it really a problem or does it open a new field of research?

Acknowledgments. We thank Manuel Güdel for his fruitful collaboration on the solar neon problem and Grégor Rauw and Arlette Noels for helpful discussions.

\section{References}

Anders, E., Grevesse, N. 1989, Geochim. Cosmochim. Acta 53, 197

Asplund, M. 2005, Ann. Rev. Astron. Astrophys. 43, 481

Asplund, M., Nordlund, A., Trampedach, R., Allende Prieto, C., Stein, R.F. 2000, A\&A 359, 729

Asplund, M., Grevesse, N., Sauval, A.J., Allende Prieto, C., Kiselman, D. 2004, A\&A 417, 751

Asplund, M., Grevesse, N., Sauval, A.J. 2005a, in "Cosmic Abundances as Records of Stellar Evolution and Nucleosynthesis", eds. T.G. Barnes III, F.N. Bash, ASP Conf. Ser. 336, 25

Asplund, M., Grevesse, N., Sauval, A.J., Allende Prieto, C., Blomme, R. 2005b, A\&A 431, 693

Basu, S., Antia, H.M. 2004, ApJ 606, L85

Drake, J.J., Testa, P. 2005, Nat. 436, 525

Grevesse, N., Noels, A. 1993, in "Origin and Evolution of the Elements", eds. N. Prantzos, E. VangioniFlam, M. Cassé, Cambridge University Press, 14

Grevesse, N., Sauval, A.J. 1998, Space Sci. Rev. 85, 161

Grevesse, N., Asplund, M., Sauval, A.J. 2005, in "Element Stratification in Stars, 40 Years of Atomic Diffusion", eds. G. Alecian, O. Richard, S. Vauclair, EAS Publ. Ser., in press

Holweger, H., Müller, E.A. 1974, Sol. Phys. 39, 19

Montalbán, J., Miglio, A., Théado, S., Noels, A., Grevesse, N. 2006, This volume, Part II

Schmelz, J.T., Nasraoui, K., Roames, J.K., Lippner, L.A., Garst, J.W. 2005, ApJ Lett., in press

Young, P.R. 2005, A\&A, in press 